\title{
Quantifying the Limits of Immunotherapies in Mice and Men
}

\author{
Liam V Brown ${ }^{\mathrm{a}, \mathrm{b}^{*}}$, Eamonn A Gaffney ${ }^{\mathrm{a}}$, Ann Ager ${ }^{\mathrm{c}}$, Jonathan Wagg ${ }^{\mathrm{d}}$, and Mark C Coles ${ }^{\mathrm{b}^{*}}$ \\ ${ }^{a}$ Wolfson Centre For Mathematical Biology, Mathematical Institute, University of Oxford \\ ${ }^{\mathrm{b}}$ Kennedy Institute of Rheumatology, University of Oxford \\ ${ }^{c}$ Systems Immunity University Research Institute and Division of Infection and Immunity, School of \\ Medicine, Cardiff University \\ ${ }^{d}$ Pharmaceutical Sciences-Clinical Pharmacology, Roche Innovation Center Basel \\ ${ }^{*}$ Corresponding authors: LVB: (last-name)@maths.ox.ac.uk.MCC: mark.coles@kennedy.ox.ac.uk
}

March 9, 2020

\begin{abstract}
Background CART-cells have demonstrated clinical success for the treatment of multiple different lymphomas and leukaemias. However, comparable clinical efficacy has not been observed for various solid tumours in human clinical trials, despite promising data from murine cancer models. Lower effective CART-cell delivery rates to human solid tumours compared to haematological malignancies in humans and solid tumours in mice might partially explain these divergent clinical outcomes, requiring the development of quantitative methods to calculate effective dosing in the clinic and models.
\end{abstract}

Methods We used anatomical and physiological data for human and rodent circulatory systems to calculate the typical value and expected variation of the perfusion of healthy and tumour tissues, and used these to estimate the upper limits of immune cell delivery rates (maximum possible under optimal conditions) across different organs, tumours types and species.

Results Estimated maximum delivery rates were up to order 10,000-fold greater in mice than humans yet reported CART-cell doses are typically only 10-100-fold lower in mice, suggesting that the effective delivery rates of CART-cells into tumours in clinical trials are far lower than in corresponding mouse models. Estimated delivery rates were compared to published PET data and found to be consistent.

Conclusions The results suggest that higher effective human doses may be needed to drive efficacy comparable to mouse solid tumour models. These increased doses raise safety and manufacturing concerns. We posit that quantitation of species and organ-specific delivery and homing of engineered T-cells will be key to unlocking their potential for solid tumours.

Keywords: Trafficking; CAR; T-cell; Immunotherapy; Failure; Mathematical; Modelling; Computational

\section{Introduction}

Cellular therapies such as CAR (Chimeric Antigen Receptor) T-cells have shown clinical efficacy against several leukaemias and lymphomas $[1,2]$. This success has not yet been matched for solid tumours, despite the efficacy seen in pre-clinical models, and a suitable dosing strategy to maximise efficacy remains uncertain [3-8]. Typical response curves (amount of CART-cell transgene observed in blood versus time) in patients with haematological disorders are marked by an initial cellular expansion (typically 100-1000-fold [9]), due to the large numbers of CART and target cells colocalising in readily accessible tissues. Expansion increases the effective cellular dose entering and proliferating within compartments with lower perfusion or less efficient access, which can drive the clearance of target cells required to achieve complete responses in these compartments. In solid tumours, relatively few target cells are in readily accessible compartments, whether due to poor perfusion or barriers to extravasation, preventing a strong initial expansion of CART-cells. Tumour regression is achieved when the rate of tumour clearance is greater than that of tumour growth, including in the least 
perfused/accessible tumour lesions. In this context, tumour clearance is a numbers game and the relative lack of success for solid tumours may be due to lower effective CART-cell doses, since the number of accessible target cells is too low to drive the early cellular expansion that, in the case of haematological malignancies, increases the effective dose.

The delivery rate of cells to different compartments of the body will likely be of importance in CART-cell or eTCR (engineered T-cell receptor) responses. For intravenous (iv) administration, cells are delivered by the circulatory system. Systematic quantitation of the variation of vascular delivery rates across organs, tumour types and species will improve understanding of comparative preclinical and clinical outcomes and inform improved dosing strategies. Physiologicallybased pharmacokinetic modelling (PBPK) has been used extensively to predict drug concentration profiles and their variability across different tissues and individuals, to estimate the efficacy of clinical dosing regimens (for recent reviews, see [10-12]). PBPK models have also been used in drug development since 2000 and are readily accepted as providing supporting information by both the US Food and Drug Administration and the European Medicines Agency. They have been further implemented in the investigation of T-cell trafficking, for example to determine the strength of the abscopal affect and influence of metastases on the primary tumour $[13,14]$ and to study localisation of adoptively transferred T-cells or cellular therapies [15-20]. However, we have not seen such models be used for quantitative exploration of the simpler consequences of differences between anatomical parameters in different species, nor an attempt to quantify and compare the maximum likely values of delivery rates of immune cells across organs and species, the aim of the present work.

We have made simple comparisons of the human, mouse and rat circulatory systems, using relevant organ, tumour and anatomical data [21-26]. We have calculated the upper bounds of cellular delivery from the circulation into each organ, considering only tissue perfusion and not factors that subsequently reduce rates of T-cell entry, such as tissuespecific extravasation probabilities or inflammation. The validity of predictions was tested through comparison to PET and imaging data taken shortly after cellular transfer, and the validity of maximum delivery rates for tumour tissue was found by comparing the typical perfusion of tumour and normal tissues. Predicted maximum delivery rates exhibited extreme differences by species. The delivery rate of cells per minute per $\mathrm{mm}^{3}$ to lungs is 20,000 -fold higher in mice than humans, yet typical doses of CART cells given to experimental mice are only 100-fold less than those in the clinic. This may partially explain the lack of success seen against solid tumours reported to date.

\section{Methods}

\subsection{Model summary}

Model: Most studies of physiologically-based pharmacokinetics (PBPK) or cellular kinetics (PBCK) make use of an ordinary differential equation (ODE) model representing the anatomy. A schematic of the anatomy appropriate for such equations is shown in Figure 1. T-cells are assumed to flow from the heart to the vasculature of different organs, where they then return or extravasate into that organ's interstitial space. Extravasated cells return to circulation via the lymphatics, except for the spleen and the pulmonary circuit, from which cells return directly. To calculate maximum delivery rates, we consider the case in which cells extravasate only in the tissue of interest, always extravasate once delivered by the vasculature, and do not return. This maximum rate is simply the rate at which cells are delivered by the vasculature, i.e. perfusion (blood flow $\boldsymbol{B}$ over total organ volume $\tilde{\boldsymbol{V}}$ ) multiplied by blood concentration $C$. More precisely,

$$
\text { Maximum delivery rate to organ } o=\frac{B_{o}}{\tilde{V}_{o}} \frac{N_{\text {tot }}}{V_{h}+\sum_{o} V_{o}},
$$

where $N_{\text {tot }}$ is the total number of cells of interest, $V_{o}$ and $\tilde{V}_{o}$ are the vascular and total volumes of organ $o$, and $V_{h}$ is the volume in the heart and interconnecting blood vessels. This expression can be shown to be equivalent to a special case of standard PBPK/PBCK models, see supplementary sections A.1 and A.1.4. This simplified scenario is presented in the inset of Figure 1. One organ is defined as the tumour bearing organ, containing a $1 \mathrm{~mm}^{3}$ tumour ('tmr') tissue volume. We consider this volume either as healthy or tumour tissue, to find how predicted delivery rates to each differ across organs and species.

\subsection{Parameter selection from literature}

Predicted T-cell delivery rates are dependent on assumed anatomical parameters (blood flow, blood volume and organ volume). We collected several anatomical reference banks from the literature [21-26], in particular the compilations by the ICRP and Shah et al [24, 26]. Each source has slightly differing fractional blood flows and volumes. To remove selection bias, delivery rates were calculated with many random values of anatomical parameters $(n=100$ per organ 


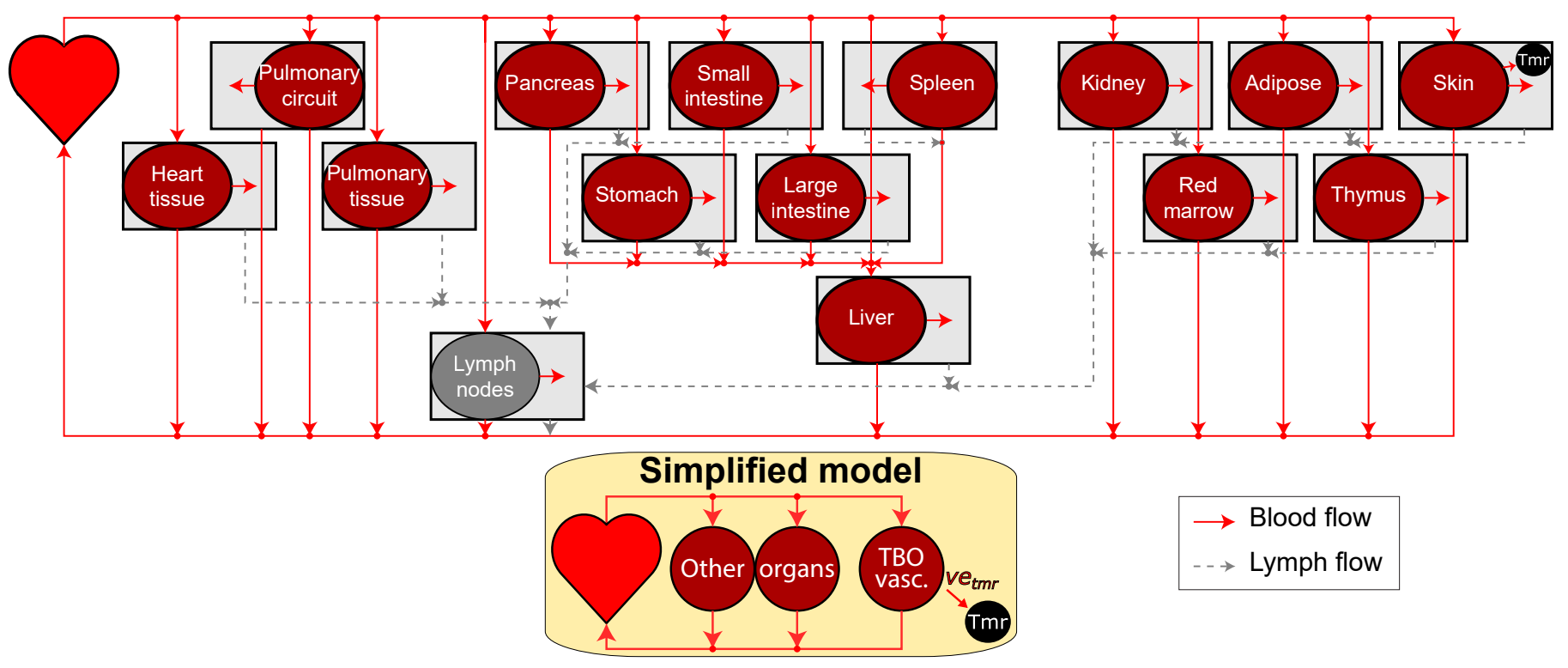

Figure 1: Model schematic: A visual summary of a model of the circulatory system. Solid and dotted lines represent blood and lymph flow respectively. Cells flow from the heart to each organ $o$, from which a proportion $e_{o}$ enters the interstitial space. Cells from the interstitium flow via the lymphatics back to the heart. One organ is designated as the tumour bearing organ (TBO), from which a proportion of cells $\nu e_{\mathrm{tmr}}$ extravasate irreversibly to the tumour ("Tmr") instead of the organ interstitium, where $\nu=\frac{V_{\text {tmr }}}{V_{\text {TBO }}}$ is the proportion of the TBO volume taken up by the tumour. The skin is set to be the tumour bearing organ as an example. Inset, below: When calculating the maximum rate of extravasation to a tumour by organ, a simplified version of the model that ignores much of the interstitial space was used, in which cells of interest are assumed not to extravasate to tissues other than the tumour. Details such as the portal vein are still present in the simplified model but not shown, for clarity.

per species), selected uniformly from the range of literature values, after which the means and standard deviations of estimated delivery rates were taken. This also serves as a proxy for population variability. To avoid using data from different studies for a single model animal, data sets that are as complete as possible were chosen. In particular, the total blood flow and blood volume, the volume of each organ, and the fractional blood flow and blood volume of each organ were recorded from each reference. These data are shown in supplementary tables S2, S3 and S4. Presented results are the mean and standard deviation of predictions obtained by choosing random values from the literature. Random parameter values are selected from the range of literature values. We cannot be more confident in any one report than another, so we choose the random values for all parameters (for each organs and species) uniformly. This process is repeated 100 times to yield the presented results. When considering tumour perfusion distinct from healthy organ perfusion, we use measurements of tumour perfusion from the literature (see supplementary table S3) and suppose that, since these are all measurements of different tumours, the data should follow a normal distribution. Thus, we choose normally distributed random values of tumour perfusion.

\subsection{Generation of presented results}

Presented data are maximum delivery rates in each species for each organ $o$, calculated using Equation 1, with some deviation due to details of the vasculature. For example, the portal vein blood flow must be added to $B_{o}$ for the liver (see supplementary section A.1 for further information). The results of Table 1 are obtained by applying data reported by Shah et al [24] to Equation 1. The results of Table 2 are obtained by multiplying the ratio of mouse to human delivery rates by the dose administered to mice, $10^{7}$.

Random results in Figure 2 are obtained by drawing uniformly random values of organ parameters $(n=100)$, calculating the maximum delivery rate with Equation 1, and subsequently finding the mean and standard deviation of delivery rates. $n=100$ values were chosen for each organ to generate an indication of delivery rate variability, whilst ensuring that the mean of selected random parameters was within $5 \%$ of the actual mean of experimental parameter values.

Random results in Figure 3 are obtained similarly, by drawing uniformly random values of organ parameters and normally 


\begin{tabular}{|lccc|ccc|}
\hline & \multicolumn{2}{c}{ Max delivery rate/(cells $\left./ \mathbf{m i n} / \mathbf{m m}^{\mathbf{3}}\right)$} & \multicolumn{3}{c|}{ BF/V/minutes } \\
Organ & Human & Mouse & Rat & Human & Mouse & Rat \\
\hline Lungs & 177.0 & $3,728,321$ & 441,221 & 5.51 & 55.4 & 63.7 \\
Kidneys & 106.7 & 266,052 & 31,767 & 3.32 & 3.95 & 4.59 \\
Thymus & 53.6 & 269,612 & 26,656 & 1.67 & 4.01 & 3.85 \\
Small intestine & 31.3 & 162,734 & 16,729 & 0.97 & 2.42 & 2.42 \\
Pancreas & 28.6 & 131,174 & 13,298 & 0.89 & 1.95 & 1.92 \\
Spleen & 27.9 & 131,336 & 13,554 & 0.87 & 1.95 & 1.96 \\
Large intestine & 22.8 & 112,344 & 11,547 & 0.71 & 1.67 & 1.67 \\
Liver (+ portal vein) & 22.5 & 113,382 & 12,310 & 0.70 & 1.68 & 1.78 \\
Heart vasculature & 22.1 & 489,648 & 31,051 & 0.69 & 7.28 & 4.49 \\
Brain & 14.4 & 49,611 & 6,007 & 0.45 & 0.74 & 0.87 \\
Lymph node & 13.0 & 29,774 & 3,064 & 0.41 & 0.44 & 0.44 \\
Stomach & 12.3 & 6,687 & 19,437 & 0.38 & 0.10 & 2.81 \\
Red marrow & 9.3 & 117,661 & 7,012 & 0.29 & 1.75 & 1.01 \\
Skin & 3.3 & 11,292 & 841 & 0.10 & 0.17 & 0.12 \\
Skeletal muscle & 1.1 & 15,537 & 1,590 & 0.03 & 0.23 & 0.23 \\
Fat & 0.8 & 13,800 & 1,419 & 0.03 & 0.21 & 0.21 \\
\hline Total blood volume / $\mathbf{m L}$ & & & & 3110 & 1.49 & 14.4 \\
\hline
\end{tabular}

Table 1: Left: predicted absolute maximum CART-cell delivery rates per volume (in cells $/ \mathrm{min} / \mathrm{mm}^{3}$ ) to non-tumour tissue in organs in humans, mice and rats, using previously compiled physiological parameter values [24]. It is assumed that organ perfusion is homogenous and $10^{8}$ CART-cells are introduced to each species. The interspecies differences in absolute delivery rates per volume depend only on organ perfusion and cell blood concentration. Right: organ perfusion (blood flow / organ volume; $\mathrm{BF} / \mathrm{V}$ ) and the total blood volume in each species, obtained by summing relevant volume data from [24]. * Note that the total blood volume from this reference is an underestimate, but it is expected to be underestimated by a similar amount in each species. The left table can be generated from the right by the formula $\frac{B}{\tilde{V}} \frac{10^{8}}{V_{\text {tot }}}$, where $B$ and $\tilde{V}$ are the organ blood flow and volume and $V_{\text {tot }}$ is the total blood volume in each species; see section 2.1 .

distributed values of tumour perfusion $P_{\mathrm{tmr}}$. The maximum delivery to tumour tissue is calculated from $P_{\mathrm{tmr}} \frac{N_{\mathrm{tot}}}{V_{h}+\sum_{o} V_{o}}$, and the maximum delivery rate to non-tumour tissue is calculated using Equation 1 for comparison. As before, $n=100$ values were chosen for each organ.

\section{Results}

\subsection{CART-cell delivery to organs in humans, mice and rats}

We calculated and compared predictions for the delivery rate per volume (cells $/ \mathrm{min} / \mathrm{mm}^{3}$ ) of a typical number of CART-cells used in the clinic $\left(10^{8}[27,28]\right)$ to non-tumour tissues in different human, rat and mouse organs. These rates are equal to the product of the organ perfusion and CART-cell blood concentration. Results calculated from a single anatomical data set ([24]) are shown in Table 1. Flow from both the hepatic artery and portal vein are included in delivery rates to the liver, and the pulmonary circuit and lung blood supply are both included for lung rates. The difference in delivery rates to the same organ in different species can be extreme, with predicted absolute lung delivery rates per volume in the mouse 21,000 times higher than in humans if the same number of CART-cells is administered to each species (obtained by dividing 3,700,000/180 from Table 1). Should a known blood concentration of endogenous cells be considered instead of a constant number, then rates per volume depend only on organ perfusion, and the absolute delivery rates for mice are up to 10 times higher than in humans. These data suggest that a more appropriate approach for scaling murine dosages to humans is to ensure that the same cellular delivery rate to tissues of interest is achieved. The results of Table 1 were used to calculate the CART-cell doses (introduced cell numbers) required to obtain the same delivery rates in humans as in mice given a typical pre-clinical dose of $10^{7}$ CART-cells. Equivalent doses are organ-specific, and most are of order $10^{10}$ to $10^{11}$ cells (Table 2).

The mean and standard deviation of predicted delivery rates obtained by random selection of anatomical parameters from all data sets [21-26] are plotted in Figure 2. To illustrate organ-specific scaling and to allow interspecies comparison 


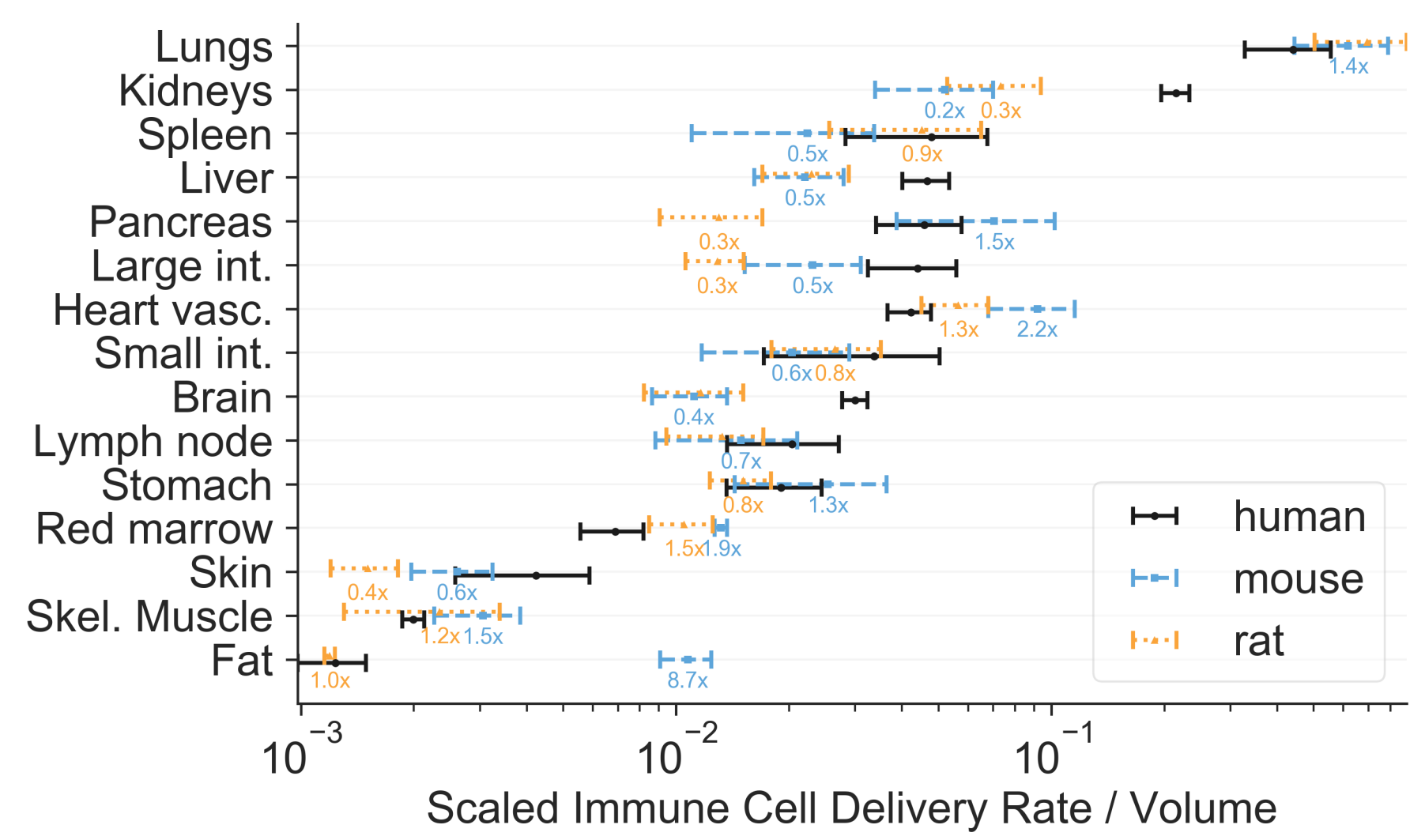

Figure 2: Relative predicted delivery rates to non-tumour tissue in organs in humans, rats and mice. Rates within each species were normalised to sum to 1.0 to give relative values for comparison. See Table 1 for a comparison of absolute rates. Predictions are presented as a mean and standard deviation over 100 repeats, with random fractional blood flows and volumes uniformly drawn from experimental data in the literature. Numerical values (text labels) give the mean predicted delivery rates in rats and mice relative to human values, with colours matching the legend. The relative distribution of rates across the major organs differs by species, and, consequently, inter-species scaling of delivery rates is organ-specific. Note that the horizontal axis is a log scale.

of the distribution of delivery rates across organs, rates are normalised such that the sum of the mean predictions within each species is 1.0. The distributions share similarities but otherwise the relative rates exhibit organ-specific scaling. For each species, the lung has the highest delivery rate, followed by the kidneys.

\subsection{CART-cell delivery to human tumours}

Predicted maximum delivery rates per $\mathrm{mm}^{3}$ of tissue described above assume that perfusion is homogeneous within a given organ. However, a tumour may have perfusion different to normal tissues. The literature was surveyed to quantify the variability of human tumour perfusion (supplementary figure S3) for incorporation into estimates of maximum delivery rates. As before, delivery rates were calculated with many random values of parameters ( $n=100$ per organ), drawn uniformly for all organ parameters and from a Gaussian distribution for tumour perfusion. The mean and standard deviation of predicted delivery rates for CART-cells to human tumours are shown in Figure 3, along with the corresponding delivery rates under the assumption of homogeneous perfusion (or equivalently, to non-tumour tissue; blue dotted boxes). The rank order of delivery rates to tumour and normal tissues are very different. In most cases, the average of predicted delivery rates for tumour tissue is similar to or less than that for normal tissue, but in some cases (e.g. the skin) it is considerably greater. However, their variation is considerable; extreme values (whiskers in the plot) vary over many orders of magnitude above and below that of the corresponding normal tissue, for most organs. 


\begin{tabular}{|lc|ll|}
\hline Organ & Equivalent dose & (continued) & \\
\hline Lungs & $1.7 \times 10^{11}$ & Heart vasculature & $2.2 \times 10^{11}$ \\
Kidneys & $2.5 \times 10^{10}$ & Brain & $3.4 \times 10^{10}$ \\
Thymus & $5.0 \times 10^{10}$ & Lymph node & $2.3 \times 10^{10}$ \\
Small intestine & $5.2 \times 10^{10}$ & Stomach & $5.4 \times 10^{09}$ \\
Pancreas & $4.6 \times 10^{10}$ & Red marrow & $1.3 \times 10^{11}$ \\
Spleen & $4.7 \times 10^{10}$ & Skin & $3.4 \times 10^{10}$ \\
Large intestine & $4.9 \times 10^{10}$ & Skeletal muscle & $1.4 \times 10^{11}$ \\
Liver & $5.0 \times 10^{10}$ & Fat & $1.7 \times 10^{11}$ \\
\hline
\end{tabular}

Table 2: Human-equivalent dosages for delivery to non-tumour tissue: The dosage of CART-cells in humans predicted to be required to give the same absolute delivery rate per $\mathrm{mm}^{3}$ as in a mouse given $10^{7}$ cells. The numbers required are much larger than many clinical dosages [27, 28].

\subsection{Maximum delivery estimates are consistent with PET imaging and radiography data}

The validity of "maximum delivery rates" to organs can be tested by comparing data from PET imaging and radiography studies in humans and rodents, in which cell localisation at early time points has been recorded. The use of an early time point is critical, as it shows the location of cells that are still in the blood or recently extravasated into an organ, before they drain back into the blood and recirculate. At later time points, localisation is a function of both cell delivery to organs and return to circulation. The delivery of radiolabelled natural killer cells from the bloodstream into individual organs has been studied in rats [29] and in human patients [30,31]. These data are presented in Figure 4 and compared to predictions from Table 1 . Patients in the human study were given $10^{8}$ to $10^{9}$ cells; the average fraction found in the liver at the first time point (30 minutes) was $8.9 \%$. This corresponds to approximately $4.5 \times 10^{7}$ cells. The rats were given $10^{6}$ to $10^{7}$ cells; the average fraction found in the liver at the first time point (30 minutes) was $23.0 \%$, or $1.2 \times 10^{6}$ cells. Adjusting the rat numbers to the human dose gives $1.2 \times 10^{8}$ cells. If we then assume a liver volume of $1700 \mathrm{ml}$ in humans and $10 \mathrm{ml}$ in rats, we obtain cell number per unit volume in the liver: $2.6 \times 10^{4}$ in humans and $1.1 \times 10^{7}$ in rats, a ratio of 429. The ratio of predicted maximum delivery rates is 546 (Table 1), 1.27-fold larger than expected from the data. Repeating this analysis for the lungs and spleen gives experimental ratios 2.0-fold less than predicted from maximum delivery rates (see Figure 4).

\section{Discussion}

\subsection{Vascular delivery and cell proliferation}

This study aimed to quantify physiological constraints on the rate of CART-cell delivery by the blood to target tissues in different species, to better predict appropriate clinical CART-cell doses from pre-clinical data. It has focused on adoptive T-cell cancer therapies, though the methodology may also apply to other therapeutic areas, including immunerelated adverse event prediction. Values were calculated assuming that $10^{8}$ T-cells are introduced; delivery rates due to any other desired number or blood concentration of cells can be calculated by multiplying results by the ratio of the desired number to $10^{8}$ or multiplying blood concentration by the total blood volume in the target species. Although models to predict expansion of a T-cell population have been studied in the past $[9,32]$, it is difficult to quantify cellular proliferation in or fractional recirculation from a given tissue. However, proliferation itself depends on exposure of transferred T-cells to their target antigen, so early responses are expected to be constrained by delivery. Several studies have established a relationship between dose and response for cellular therapies, despite proliferation increasing the effective dose over time [28, 33-35]. Furthermore, delivery of cells that proliferate outside of a given tumour site would also be constrained by vascular delivery. The maximum rate of delivery due to the anatomy can be estimated with greater confidence and wider applicability than can an estimated time-course of T-cell concentration that considers proliferation and contraction, so proliferation was not considered in this work and will be the focus of future studies.

\subsection{Organ-specific delivery rates and their variation}

Results predict that the highest CART-cell delivery rates are in organs with the highest perfusion: the lungs and kidneys in humans (Figure 2). When measurements of tumour-specific perfusion are considered (Figure 3), it is the kidneys, skin, large intestine and lungs that are predicted to have the highest delivery rates per $\mathrm{mm}^{3}$, consistent with non-cellular 


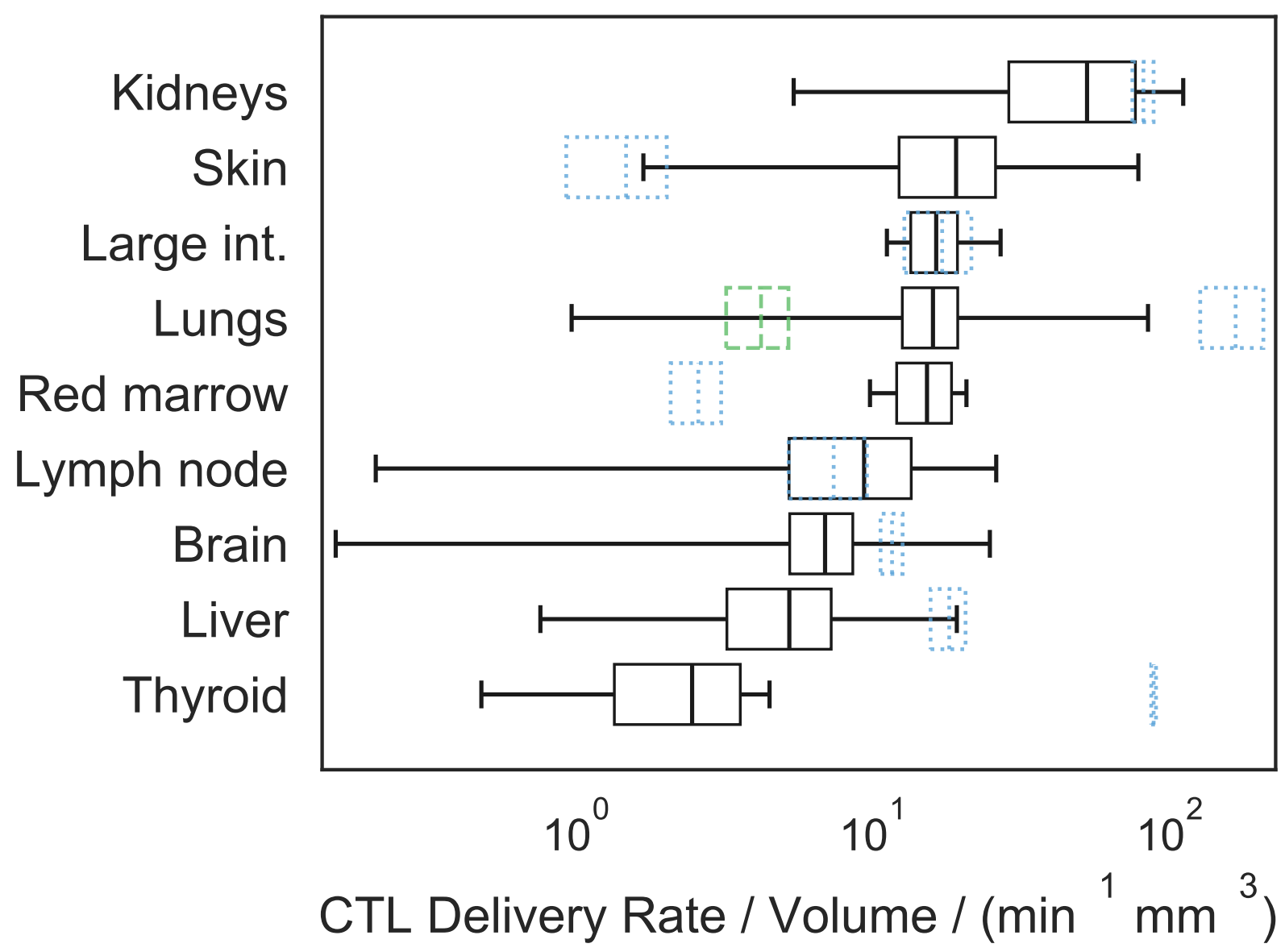

Figure 3: Absolute predicted delivery rates to human tumours, compared to non-tumour tissue, assuming $10^{8}$ CART-cells are administered IV. Predictions are presented as a mean and standard deviation over 100 repeats, with random anatomical parameters and tumour perfusion drawn from experimental data in the literature. Black boxes represent the mean and standard deviation of predicted tumour delivery rates, and whiskers indicate predictions using the extremes of possible tumour perfusion according to the literature. Blue dotted boxes indicate the mean and standard deviation of predicted delivery to non-tumour tissue, i.e. the data used to generate Figure 2. The green dashed box indicates delivery rates per $\mathrm{mm}^{3}$ to healthy lung tissue when the pulmonary circuit is assumed not to contribute. Note that now the kidneys and skin have the highest predicted tumour delivery rates, and that the horizontal axis is a log scale.

immunotherapies (IL-2 and checkpoint blockade) having the highest efficacy in kidney, skin, colon and lung tumours [36-41], and the hypothesis that efficacy is driven in part by tissue perfusion. For cellular therapies including CARTcells, vascular delivery should similarly correlate with efficacy, with the additional factor that T-cells must extravasate into target tissues. Both naïve and ex vivo T-cells preferentially extravasate into lymph nodes, spleen and liver [42-44], consistent with CART-cell efficacy in haematological disorders but not solid tumours $[1,27,28]$. Tumour tissue may be vascular or avascular, and inflamed or uninflamed, meaning that predicted 'maximum' delivery rates for normal tissues may not be applicable to tumours. However, predicted mean delivery rates into tumours exceed those to normal tissue for only a minority of organs (Figure 3), including the skin. Predicted delivery rates to tumours in the skin vary over many orders of magnitude but are usually greater than those for normal tissue. Healthy skin is not usually highly perfused and contains shunts to control blood flow in response to temperature. Most anatomical data for the skin describes the organ at rest and at room temperature with no inflammation, meaning most shunts will be open. Tumour tissue can increase its perfusion through inflammation or angiogenesis and likely subverts these shunts, which could explain the greater mean and variation in predicted delivery rates for skin tumours. Liver and kidney tissues are highly perfused at rest, which are unlikely to be improved by random tumour angiogenesis; accordingly, predicted delivery rates to tumours in these organs do not exceed normal tissue. Predictions for red bone marrow indicate that 
a)

Human

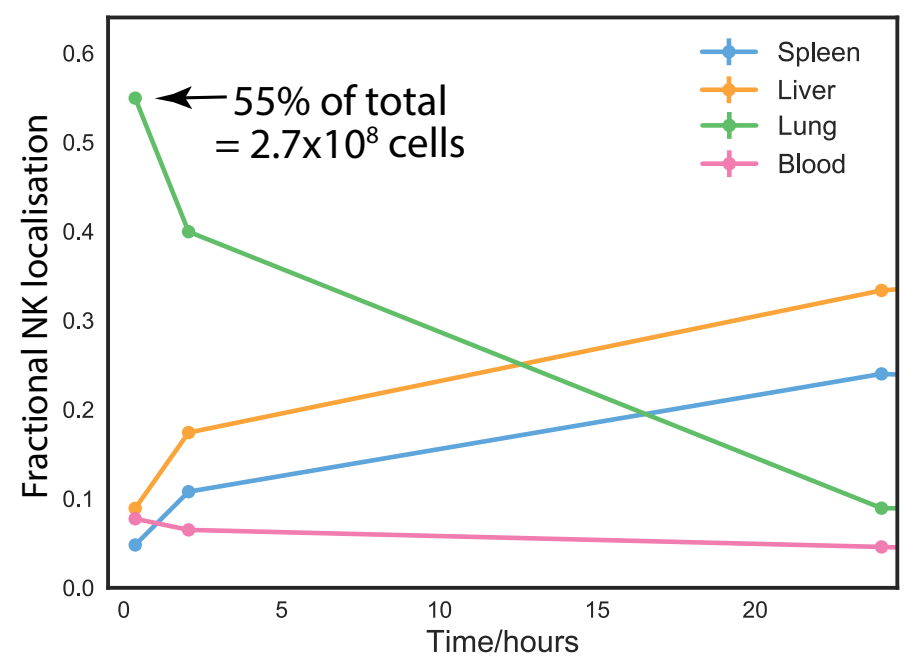

Rat

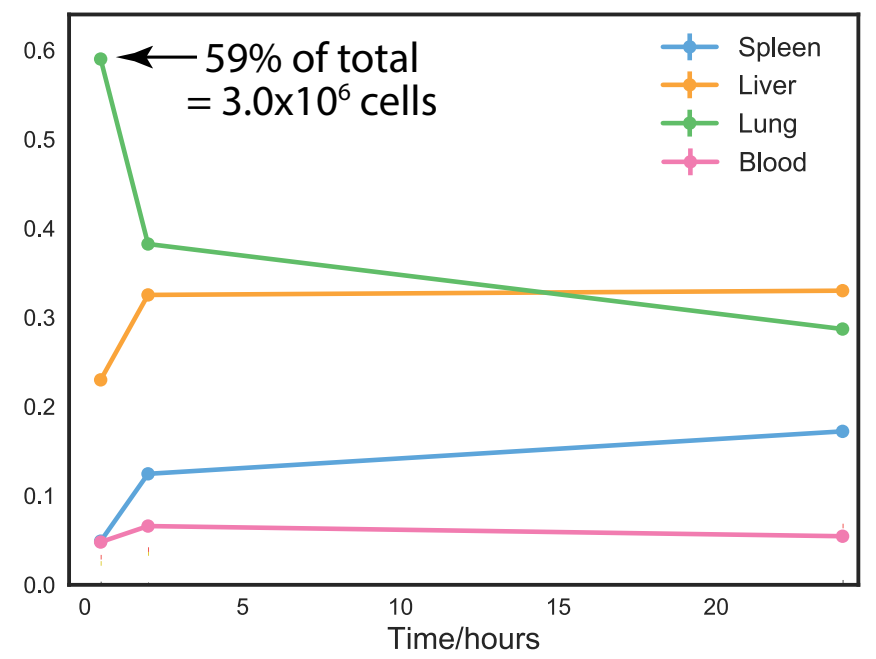

b)

\begin{tabular}{|l|cc|cc|cc|}
\hline & \multicolumn{2}{|c|}{ Lung } & \multicolumn{2}{c|}{ Liver } & \multicolumn{2}{c|}{ Spleen } \\
& Human & Rat & Human & Rat & Human & Rat \\
\hline Organ fraction after 30 mins & $55.0 \%$ & $59.0 \%$ & $8.9 \%$ & $23.0 \%$ & $4.8 \%$ & $4.9 \%$ \\
Number of cells in organ & $2.7 \times 10^{8}$ & $3.0 \times 10^{6}$ & $4.5 \times 10^{7}$ & $1.2 \times 10^{6}$ & $2.4 \times 10^{7}$ & $2.5 \times 10^{5}$ \\
Number scaled by dose & $2.7 \times 10^{8}$ & $3.0 \times 10^{8}$ & $0.45 \times 10^{8}$ & $1.2 \times 10^{8}$ & $0.24 \times 10^{8}$ & $0.25 \times 10^{8}$ \\
Number of cells / organ volume & $2.4 \times 10^{5}$ & $3.0 \times 10^{8}$ & $2.6 \times 10^{4}$ & $1.1 \times 10^{7}$ & $1.7 \times 10^{5}$ & $4.1 \times 10^{7}$ \\
Ratio of rat to human & \multicolumn{2}{|c|}{1226} & \multicolumn{2}{|c|}{429} & \multicolumn{2}{c|}{245} \\
Ratio of predicted delivery rates & \multicolumn{2}{|c|}{2437} & \multicolumn{2}{|c|}{546} & \multicolumn{2}{c|}{485} \\
Delivery ratio/localisation ratio & \multicolumn{2}{|c|}{1.99} & \multicolumn{2}{|c|}{1.27} & \\
\hline
\end{tabular}

Figure 4: Comparison of reported localisation of radiolabelled natural killer (NK) cells in rats and humans to predicted maximum delivery rates [29-31]. a) Reproductions of the reported data, after normalising data at each time point such that the total radioactivity (localisation) is 1.0 at all time points. Annotations indicate the initial count of cells in the lung in each species. b) Analysis of the data. The dosage and fractional localisation in each organ can be used to calculate the number of NK cells present in each organ at each time point. By accounting for the different dose given to each species and choosing an appropriate estimate for organ volumes in each species, the number of cells per volume in each species can be calculated. The rat/human ratio of the number of cells in each organ can be compared to the ratio of predicted maximum delivery rates per volume, obtained from Table 1.

tumour perfusion can greatly outstrip normal tissue perfusion. Though surprising, the bone red bone marrow result is consistent with studies in which bone perfusion was measured in healthy control bone and tumour sites in patients with bone cancers and metastases [45]. Finally, predicted delivery rates to lung tumours may or may not exceed that of normal tissue, depending on whether the pulmonary circuit is assumed to contribute to tumour blood supply (blue dotted box) or not (green dashed box). Aside from these exceptions, results suggest that predicted maximum delivery rates to normal tissue are greater than those to tumour tissue of the same origin in most cases, and so appropriate to use as a guideline to compare species.

Both figures 2 and 3 show that predicted delivery rates are highly variable, which may be caused by differences in experimental techniques or individual variation. Physiological differences and behaviour both impact blood flow distributions; blood flow to the mesentery increases after a meal, muscles during exercise, or the skin in response to temperature. This effect is utilised in the clinic to prevent hair loss in chemotherapy patients by cooling the scalp. CART-cell therapies could be targeted to organs such as the mesentery or skin through meal consumption or temperature control, and tumour-specific blood flow could be increased with vessel normalisation associated with anti-angiogenic therapies (e.g. Avastin). Both human and rodent anatomical parameters vary, impacting any results that depend on anatomical parameters. If variability is not captured and/or care is not taken to control factors that alter blood flows (e.g. anaes- 
thesia, exercise or the time of day [46]), then comparison of data sets may be invalid. Ideally, any study making use of blood flows and organ volumes should consider multiple measurements and include 'error' bars to indicate variation.

\subsection{Species-specific delivery rates and dosage scaling}

Relative delivery rates are distributed differently across organs in each species, meaning that dose scaling is organ-specific (Figure 2, Table 1). Predicted absolute delivery rates of the same dose of CART-cells $\left(10^{8}\right)$ exhibited surprisingly extreme differences between species, with delivery per unit tissue volume to mouse lungs 21,000 times higher than in humans, largely because of the difference in total blood volumes between mice $(2 \mathrm{~mL})$ and humans $(5 \mathrm{~L})$. To test the relevance of these "maximum delivery rates" and validate the model, we analysed published PET imaging and radiography studies of natural killer (NK) cells in humans and rats [29-31] and calculated the cell numbers present in various organs at early time points (Section 3.3). The human/rat ratios of NK unit volume in the lungs, liver and spleen 30 minutes after infusion were compared to the human/rat ratios of predicted maximum delivery rates. The measured localisation ratios are 1.3 to 2.0-fold greater than predictions for delivery rate ratios. Such small discrepancies are not unexpected, as delivery rate ratios would only equal localisation ratios if the blood concentration of NK cells and hence delivery rates were constant. However, the earliest experimental time point is 30 minutes, providing sufficient time for blood recirculation (as cardiac output/minute is greater than total blood volume in humans and rats). The rates of extravasation and return in each organ may differ between humans and rats, and the experimental technique and total amount of radioactivity at the first time point differs between the two studies. Regardless of these potentially confounding factors, the observations are consistent with predictions.

Despite the considerably greater delivery rates of cells in mice than humans, typical doses (cell numbers) introduced to mice are not considerably lower than those given to humans. Most patients are given CART-cell dosages between $10^{7}$ and $10^{9}$ cells $[27,28]$, whilst mouse studies have used (for example) two doses of 1 to $2.5 \times 10^{6}$ cells a week apart [6], two doses of $10^{7}$ cells a week apart [3], and a single dose of $10^{7}$ cells [4]. To illustrate how large these doses are, we calculated equivalent human dosages that would yield the same absolute delivery rates in humans as in a mouse given $10^{7}$ CART-cells (Table 2). The resulting doses range between $10^{10}$ and $10^{11} \mathrm{~T}$-cells, much higher than typical clinical doses and many dose escalation studies [28]. This may explain why pre-clinical success does not always translate to the clinic. A pre-clinical study of a CEA CART-cell therapy resulted in regression of subcutaneous tumours in mice with a dose of $5 \times 10^{6}$ cells (equivalent to $1.7 \times 10^{10}$ in humans) [47]. In another study, a CART-cell therapy restricted the growth of pancreatic tumours in all treated mice to below the limit of detection with a dose of $10^{7}$ cells (equivalent to $4.6 \times 10^{10}$ in humans) [48]. A study in which lower doses of around $10^{5}$ anti-CD19 cells (human equivalent, using total blood volume only, of $2 \times 10^{8}$ cells) were given to mice as a 'stress-test' was associated with poor tumour control [35]. In the clinic, a study of CEA CART-cells against colorectal cancer [34] escalated doses between $10^{7}$ and $10^{10}$ cells. The authors found that the lower doses did not stop tumour progression (in 3 of 14 of presented patients) and higher doses achieved only stable disease. Our results suggest that dosages of order $10^{10}$ cells would be required to drive tumour regression at the primary site, and $10^{11}$ would be required for the lung metastases. Clinical studies in which Tumour Infiltrating Lymphocytes (TILs) were introduced in greater numbers $\left(10^{9}\right.$ to $\left.10^{11}\right)$ [49-52] and in which CART-cells were introduced regionally (bypassing trafficking via the bloodstream) [27] are associated with greater efficacy. An important caveat of these simple comparisons is that some of the studies lymphodepleted the mice or patients before infusing T-cells, which aids proliferation, and some did not.

In patients with advanced metastatic disease, CART-cell dosage must be sufficient to drive tumour regression at the least perfused and/or the fastest growing site. To avoid dosage-linked increases in adverse events such as cytokine release or encephalopathy syndromes, methods to increase the effective dose on-site and not elsewhere should be considered, including alternate modes of administration, triggering proliferation at sites of interest, coadministration of inhibitors (e.g. anti-IL6), or interventions to alter blood flows described in Section 4.2.

We used natural killer cell localisation data to validate the model, by confirming that early localisation of cells correlates with predicted maximum delivery rates and assuming that natural killer and T-cells behave similarly to each other at short time scales. A more appropriate validation would compare predictions to the localisation of adoptively transferred cells to solid tumours in mice and humans, however, such data is sparsely published and we have found no reported data for humans that includes organ and tumour localisation at an early time point (of the order of minutes). Such data would be useful for further work, as would a time course that could be used to quantify the subsequent constraints imposed by homing and proliferation of cells.

The numbers presented here compare organs like-for-like between mice and humans, but scaling is more uncertain for xenografts. The ratio of the maximum delivery rate per volume to skin tissue between mice and humans is 2 if the same blood concentration of immune cells is assumed, or 3400 if the same number of immune cells is assumed (calculated 
from Table 1). The ratio of delivery rates per volume to mouse skin versus human kidney tissue, meanwhile, is 0.05 if the same concentration of cells is used, or 100 if the same number of immune cells is assumed. A previous study [53] has shown that small xenografts have similar local perfusion to the original tissue, but larger xenografts have reduced perfusion relative to the original tissue. This non-linearity further confounds extrapolation of preclinical results. This highlights some of the historically observed difficulties in the clinical translation of preclinical mouse xenograft model results [54]. In addition to consideration of physiological and immunological differences (such as the adhesion molecules required for T-cell extravasation), interpretation of pre-clinical therapeutic success requires dosages to be appropriately scaled to humans. A model that considers organ-specific blood flow and volumes across species can be used to more precisely estimate likely efficacious human doses.

\subsection{Prediction refinement by T-cell homing and further considerations}

The presented results are the predicted maximum delivery rates of CART-cells per unit volume (cells $/ \mathrm{min} / \mathrm{mm}^{3}$ ) to organs and tumours, based on only organ blood flows and volumes. Refining these predictions requires quantification of CART-cell proliferation and organ-specific homing. The probability of T-cell extravasation differs by location and cell type. Naïve T-cells extravasate mainly into the lymph nodes or spleen and activated cells have a higher probability of extravasating into non-lymphoid tissues [42, 55], distributed according to upregulated homing receptors (e.g. Lselectin or CCR7 [56]). These probabilities may differ across species (e.g. homing receptor CXCR1 is present in humans but not mice [57]), further limiting inter-species extrapolation of pre-clinical results. Homing receptor density, vessel normalisation and hence homing probabilities may further differ in tumour tissue, particularly following therapies such as Avastin [58, 59]. It is possible to quantify organ-specific homing by fitting ODE models (like the model shown in supplementary section A.1) to T-cell localisation data in experimental animals, as previous authors have done, e.g. [19]. However, we have found very limited equivalent human data for cross-species comparison, which is the primary aim of this work. Parameters obtained from fits to multiple experiments would differ due to differences in the animals and the cells, so several datasets would be required to quantify the variation of and/or a confidence interval for parameter estimates. The focus of this study is on anti-tumour therapies, where tumour homing would be affected by factors such as inflammation. For this reason, we chose to quantify maximum delivery rates by examining the case where T-cells have a $100 \%$ probability of extravasation in the target organ, and no extravasation elsewhere. Species comparisons are made by implicitly assuming that homing probabilities in a given organ or tumour would be similar between species. Expected variation in predictions was quantified by using the variation among anatomical reference values as a proxy. Both maximum values and this variation could be improved by more precise measurements of blood flows and volumes using the same techniques in each species, or else finding anatomical parameters for a precise experimental animal of interest.

Another challenge for CART-cells in solid tumours is the identification of suitable target antigen. The ideal antigen is highly expressed on tumour cells and not expressed on healthy cells elsewhere. A typical target for B-cell malignancies is CD19 [28], as it is expressed by the entire pool of B-cells and is limited almost exclusively to B-cells. Several different antigens have been targeted for solid tumours, but with limited success (for example, GD2 has had encouraging results [28]). Target antigen may only be expressed by a subset of tumour cells and may not be sufficiently rare elsewhere in the body. For example, CAIX is expressed in some renal cell carcinomas, but it is also expressed in the liver bile duct resulting in on-target, off-tumour toxicities in a phase III trial [60]. Tumours may evolve to reduce expression of target antigen in response to successful T-cell killing, reducing the rate of tumour elimination or promoting outgrowth of therapy-resistant cells. Although these considerations are a barrier to treatment success, the rate at which cells can be delivered is a parallel and important factor. CART-cells that are specific for an antigen that is expressed on most tumour cells will not drive tumour regression if their kill rate is lower than the tumour growth rate, given the combined rates of T-cell delivery and proliferation. On the other hand, CART-cells specific for a rarer antigen may drive tumour regression if they arrive in sufficient numbers to eliminate all cells carrying that antigen, subsequently proliferating to greater numbers to drive regression at more restricted sites and/or drive a secondary response against one or more other antigens (i.e. epitope spread). Like T-cell delivery rates and T-cell extravasation probabilities, typical tumour growth rates are species, organ and individual specific. Together, these considerations show that tumour immunotherapy is a numbers game and hence more generally quantitative studies can be a useful tool for understanding the translational gap between pre-clinical and clinical outcomes. 


\section{Conclusions}

Details of the human, rat and mouse circulatory systems were considered to predict CART-cell delivery to human tumours, and to human, rat and mouse organs. Predictions show up to an order of 10,000-fold increased CART-cell delivery per unit volume of target tissue in mice than humans, while typical clinical cell therapy dosages are 100-fold less than typical pre-clinical doses. These numbers are consistent with experimental studies of NK cell localisation and various clinical observations. These predictions could partially explain why pre-clinical models of solid tumour clearance by CART-cells show greater efficacy than in humans. Dosage scaling was found to be organ-specific and is particularly hard to quantify for xenografts, confounding the interpretation of pre-clinical results and lowering their potential clinical value, which is an important consideration in the context of the reduction and replacement of animal experiments. Control of tumour and organ-specific blood flow through exercise, circadian timing or food consumption could increase cellular delivery to tumour sites without raising the prospect of adverse outcomes, while vascular normalisation may also induce such benefits, though with accompanying risk. More generally, cellular kinetic and dynamic models will lead to better understanding of how pre-clinical outcomes translate to the clinic, and hence better determination of appropriate clinical dosages and treatment strategies for cell-based therapies.

\section{Declarations}

Availability of data and code: Code and parameter data for humans, mice and rats used to generate the results of this work are available as supplementary materials.

Author contributions: LVB designed the model code, did the analysis and wrote the manuscript. All other authors supervised, advised and edited the manuscript.

Competing interests: JW is an employee and shareholder of Hoffmann-La Roche. LVB has previously completed an internship at that same company.

Funding: This research was supported by funding from a Clarendon Scholarship, Hoffmann-La Roche and the Engineering and Physical Sciences Research Council (EPSRC), grant number EP/L016044/1.

\section{References}

[1] David Pettitt, Zeeshaan Arshad, James Smith, Tijana Stanic, Georg Holländer, and David Brindley. CAR-T Cells: A Systematic Review and Mixed Methods Analysis of the Clinical Trial Landscape. Molecular Therapy, 26(2): 342-353, 2018. doi: 10.1016/j.ymthe.2017.10.019.

[2] Charlotte Graham, Rebecca Hewitson, Antonio Pagliuca, and Reuben Benjamin. Cancer immunotherapy with CAR-T cells - Behold the future. Clinical Medicine, Journal of the Royal College of Physicians of London, 18(4): 324-328, 2018. ISSN 14734893. doi: 10.7861/clinmedicine.18-4-324.

[3] He Li, Yao Huang, Du-Qing Jiang, Lian-Zhen Cui, Zhou He, Chao Wang, Zhi-Wei Zhang, Hai-Li Zhu, Yong-Mei Ding, Lin-Fang Li, Qiang Li, Hua-Jun Jin, and Qi-Jun Qian. Antitumor activity of EGFR-specific CAR T cells against non-small-cell lung cancer cells in vitro and in mice. Cell Death $\mathscr{E}$ Disease, 9(2):177, 2018. ISSN 2041-4889. doi: 10.1038/s41419-017-0238-6.

[4] Nobuhiro Nishio, Iulia Diaconu, Hao Liu, Vincenzo Cerullo, Ignazio Caruana, Valentina Hoyos, Lisa BouchierHayes, Barbara Savoldo, and Gianpietro Dotti. Armed oncolytic virus enhances immune functions of chimeric antigen receptor-modified T cells in solid tumors. Cancer Research, 74(18):5195-5205, 2014. ISSN 15387445. doi: 10.1158/0008-5472.CAN-14-0697.

[5] Kheng Newick, Edmund Moon, and Steven M. Albelda. Chimeric antigen receptor T-cell therapy for solid tumors. Molecular Therapy - Oncolytics, 3(October 2015):16006, 2016. ISSN 23727705. doi: 10.1038/mto.2016.6.

[6] Mauro P. Avanzi, Oladapo Yeku, Xinghuo Li, Dinali P. Wijewarnasuriya, Dayenne G. van Leeuwen, Kenneth Cheung, Hyebin Park, Terence J. Purdon, Anthony F. Daniyan, Matthew H. Spitzer, and Renier J. Brentjens. Engineered Tumor-Targeted T Cells Mediate Enhanced Anti-Tumor Efficacy Both Directly and through Activation of the Endogenous Immune System. Cell Reports, 23(7):2130-2141, 2018. ISSN 22111247. doi: 10.1016/j.celrep.2018.04.051. 
[7] Jeffrey R. Sachs, Kapil Mayawala, Satvik Gadamsetty, Soonmo Peter Kang, and Dinesh P. de Alwis. Optimal Dosing for Targeted Therapies in Oncology: Drug Development Cases Leading by Example. Clin. Cancer Res., 22 (6):1318-1324, Mar 2016. ISSN 1078-0432. doi: 10.1158/1078-0432.CCR-15-1295.

[8] Yan Ji, Jin Y. Jin, David M. Hyman, Geoffrey Kim, and Ajit Suri. Challenges and Opportunities in Dose Finding in Oncology and Immuno-oncology. Clin. Transl. Sci., 11(4):345, Jul 2018. doi: 10.1111/cts.12540.

[9] Andrew M. Stein, Stephan A. Grupp, John E. Levine, Theodore W. Laetsch, Michael A. Pulsipher, Michael W. Boyer, Keith J. August, Bruce L. Levine, Lori Tomassian, Sweta Shah, Mimi Leung, Pai-Hsi Huang, Rakesh Awasthi, Karen Thudium Mueller, Patricia A. Wood, and Carl H. June. Tisagenlecleucel Model-Based Cellular Kinetic Analysis of Chimeric Antigen Receptor-T Cells. CPT Pharmacometrics Syst. Pharmacol., 8(5):285-295, May 2019. ISSN 2163-8306. doi: 10.1002/psp4.12388.

[10] K Yoshida, N Budha, and J. Jin. Impact of Physiologically Based Pharmacokinetic Models on Regulatory Reviews and Product Labels: Frequent Utilization in the Field of Oncology. Clinical Pharmacology and Therapeutics, 101 (5):597-602, 2017. doi: 10.1002/cpt.622.

[11] Teerachat Saeheng, Kesara Na-Bangchang, and Juntra Karbwang. Utility of physiologically based pharmacokinetic (PBPK) modeling in oncology drug development and its accuracy: a systematic review. European Journal of Clinical Pharmacology, July 2018. ISSN 0031-6970. doi: 10.1007/s00228-018-2513-6.

[12] Mohamad Shebley, Punam Sandhu, Arian Emami Riedmaier, Masoud Jamei, Rangaraj Narayanan, Aarti Patel, Sheila Annie Peters, Venkatesh Pilla Reddy, Ming Zheng, Loeckie Zwart, Maud Beneton, Francois Bouzom, Jun Chen, Yuan Chen, Yumi Cleary, Christiane Collins, Gemma L. Dickinson, Nassim Djebli, Heidi J. Einolf, Iain Gardner, Felix Huth, Faraz Kazmi, Feras Khalil, Jing Lin, Aleksandrs Odinecs, Chirag Patel, Haojing Rong, Edgar Schuck, Pradeep Sharma, Shu-Pei Wu, Yang Xu, Shinji Yamazaki, Kenta Yoshida, and Malcolm Rowland. Physiologically based pharmacokinetic model qualification and reporting procedures for regulatory submissions: A consortium perspective. Clinical Pharmacology \& Therapeutics, 104(1):88-110, 2018. doi: 10.1002/cpt.1013.

[13] Jan T. Poleszczuk, Kimberly A. Luddy, Sotiris Prokopiou, Mark Robertson-Tessi, Eduardo G. Moros, Mayer Fishman, Julie Y. Djeu, Steven E. Finkelstein, and Heiko Enderling. Abscopal benefits of localized radiotherapy depend on activated T-cell trafficking and distribution between metastatic lesions. Cancer Research, 76(5):10091018, 2016. ISSN 15387445. doi: 10.1158/0008-5472.CAN-15-1423.

[14] Rachel Walker, Jan Poleszczuk, Shari Pilon-Thomas, Sungjune Kim, Alexander A.R.A. Anderson, Brian J. Czerniecki, Louis B. Harrison, Eduardo G. Moros, and Heiko Enderling. Immune interconnectivity of anatomically distant tumors as a potential mediator of systemic responses to local therapy. Scientific Reports, 8(1):1-11, 2018. ISSN 20452322. doi: 10.1038/s41598-018-27718-1.

[15] Hui Zhu, Robert J Melder, Laurence T Baxter, and Rakesh K Jain. Physiologically Based Kinetic Model of Effector Cell Biodistribution in Mammals: Implications for Adoptive Immunotherapy. (617):3771-3781, 1996.

[16] Robert J Melder, Lance L Munn, Brian R Stoll, Edgardo M Marecos, Laurence T Baxter, Ralph Weissleder, and Rakesh K Jain. Systemic distribution and tumor localization of adoptively transferred lymphocytes in mice: comparison with physiologically based pharmacokinetic model. Neoplasia, 4(1):3-8, 2002. ISSN 1522-8002. doi: $10.1038 / \mathrm{sj} / \mathrm{neo} / 7900209$.

[17] Stuart W. Friedrich, Stephany C. Lin, Brian R. Stoll, Laurence T. Baxter, Lance L. Munn, and Rakesh K. Jain. Antibody-directed effector cell therapy of tumors: Analysis and optimization using a physiologically based pharmacokinetic model. Neoplasia, 4(5):449-463, 2002. ISSN 15228002. doi: 10.1038/sj.neo.7900260.

[18] Antari Khot, Satoko Matsueda, Veena A. Thomas, Richard C. Koya, and Dhaval K. Shah. Measurement and Quantitative Characterization of Whole-Body Pharmacokinetics of Exogenously Administered T Cells in Mice. $J$. Pharmacol. Exp. Ther., 368(3):503-513, Mar 2019. ISSN 0022-3565. doi: 10.1124/jpet.118.252858.

[19] Vitaly V. Ganusov and Jeremy Auerbach. Mathematical Modeling Reveals Kinetics of Lymphocyte Recirculation in the Whole Organism. PLoS Comput. Biol., 10(5):e1003586, May 2014. ISSN 1553-7358. doi: 10.1371/journal.pcbi. 1003586 . 
[20] Hulin Wu, Arun Kumar, Hongyu Miao, Jeanne Holden-Wiltse, Timothy R. Mosmann, Alexandra M. Livingstone, Gabrielle T. Belz, Alan S. Perelson, Martin S. Zand, and David J. Topham. Modeling of Influenza-Specific CD8+ $\mathrm{T}$ Cells during the Primary Response Indicates that the Spleen Is a Major Source of Effectors. J. Immunol., 187 (9):4474-4482, Nov 2011. ISSN 0022-1767. doi: 10.4049/jimmunol.1101443.

[21] R P Brown, M D Delp, S L Lindstedt, L R Rhomberg, and R P Beliles. Physiological parameter values for physiologically based pharmacokinetic models. Toxicology and Industrial Health, 13(4):407-84, 1997. ISSN 07482337. doi: $10.1177 / 074823379701300401$.

[22] John F. Graf, Bernhard J. Scholz, and Maria I. Zavodszky. BioDMET: A physiologically based pharmacokinetic simulation tool for assessing proposed solutions to complex biological problems. Journal of Pharmacokinetics and Pharmacodynamics, 39(1):37-54, 2012. ISSN 1567567X. doi: 10.1007/s10928-011-9229-x.

[23] L T Baxter, H Zhu, D G Mackensen, and R K Jain. Physiologically based pharmacokinetic model for specific and nonspecific monoclonal antibodies and fragments in normal tissues and human tumor xenografts in nude mice. Cancer Res, 54(6):1517-1528, 1994. ISSN 0008-5472.

[24] Dhaval K. Shah and Alison M. Betts. Towards a platform PBPK model to characterize the plasma and tissue disposition of monoclonal antibodies in preclinical species and human. Journal of Pharmacokinetics and Pharmacodynamics, 39(1):67-86, 2012. ISSN 1567567X. doi: 10.1007/s10928-011-9232-2.

[25] Sheila Annie Peters. Physiologically-Based Pharmacokinetic (PBPK) Modeling and Simulations: Principles, Methods, and Applications in the Pharmaceutical Industry. Wiley, 2012. ISBN 978-0-470-48406-7.

[26] International commission on radiological protection. Basic Anatomical and Physiological Data for Use in Radiological Protection Reference Values. ICRP Publication 89, Ann. ICRP, 32(3-4), 2002.

[27] Praveen Sridhar and Fabio Petrocca. Regional delivery of chimeric antigen receptor (CAR) T-cells for cancer therapy. Cancers, 9(7):1-10, 2017. ISSN 20726694. doi: 10.3390/cancers9070092.

[28] Jessica Hartmann, Martina Schüßler-Lenz, Attilio Bondanza, and Christian J Buchholz. Clinical development of CAR T cells - challenges and opportunities in translating innovative treatment concepts. EMBO Molecular Medicine, 9(9):e201607485, 2017. ISSN 1757-4676. doi: 10.15252/emmm.201607485.

[29] B. Rolstad, R. B. Herberman, and C. W. Reynolds. Natural killer cell activity in the rat. V. The circulation patterns and tissue localization of peripheral blood large granular lymphocytes (LGL). J. Immunol., 136(8):2800-2808, Apr 1986. ISSN 0022-1767. URL https://www.ncbi.nlm.nih.gov/pubmed/3485674.

[30] J.-M. Brand, B. Meller, K. Von Hof, J. Luhm, M. Bähre, H. Kirchner, and C. Frohn. Kinetics and organ distribution of allogeneic natural killer lymphocytes transfused into patients suffering from renal cell carcinoma. Stem Cells Dev., 13(3):307-314, Jun 2004. ISSN 1547-3287. doi: 10.1089/154732804323099235.

[31] Birgit Meller, Christoph Frohn, Jörg-Matthias Brand, Isabel Lauer, Lutz F. Schelper, Katharina von Hof, Holger Kirchner, Eckart Richter, and Manfred Baehre. Monitoring of a new approach of immunotherapy with allogenic (111)In-labelled NK cells in patients with renal cell carcinoma. Eur. J. Nucl. Med. Mol. Imaging, 31(3):403-407, Mar 2004. ISSN 1619-7070. doi: 10.1007/s00259-003-1398-4.

[32] Julien Vibert and Véronique Thomas-Vaslin. Modelling T cell proliferation: Dynamics heterogeneity depending on cell differentiation, age, and genetic background. PLoS Computational Biology, 13(3):1-25, 2017. ISSN 15537358. doi: $10.1371 /$ journal.pcbi.1005417.

[33] Luca Gattinoni, Christopher A. Klebanoff, Douglas C. Palmer, Claudia Wrzesinski, Keith Kerstann, Zhiya Yu, Steven E. Finkelstein, Marc R. Theoret, Steven A. Rosenberg, and Nicholas P. Restifo. Acquisition of full effector function in vitro paradoxically impairs the in vivo antitumor efficacy of adoptively transferred CD8+ T cells. Journal of Clinical Investigation, 115(6):1616-1626, 2005. ISSN 00219738. doi: 10.1172/JCI24480.

[34] Chengcheng Zhang, Zhe Wang, Zhi Yang, Meiling Wang, Shiqi Li, Yunyan Li, Rui Zhang, Zhouxing Xiong, Zhihao Wei, Junjie Shen, Yongli Luo, Qianzhen Zhang, Limei Liu, Hong Qin, Wei Liu, Feng Wu, Wei Chen, Feng Pan, Xianquan Zhang, Ping Bie, Houjie Liang, Gabriele Pecher, and Cheng Qian. Phase I Escalating-Dose Trial of CAR-T Therapy Targeting CEA+Metastatic Colorectal Cancers. Molecular Therapy, 25(5):1248-1258, 2017. ISSN 15250024. doi: 10.1016/j.ymthe.2017.03.010. 
[35] Zeguo Zhao, Maud Condomines, Sjoukje J. C. van der Stegen, Fabiana Perna, Christopher C. Kloss, Gertrude Gunset, Jason Plotkin, and Michel Sadelain. Structural Design of Engineered Costimulation Determines Tumor Rejection Kinetics and Persistence of CAR T Cells. Cancer Cell, 28(4):415-428, Oct 2015. ISSN 1535-6108. doi: 10.1016/j.ccell.2015.09.004.

[36] Yanping Xiao and Gordon J. Freeman. The microsatellite instable subset of colorectal cancer is a particularly good candidate for checkpoint blockade immunotherapy. Cancer Discovery, 5(1):16-18, 2015. ISSN 21598290. doi: 10.1158/2159-8290.CD-14-1397.

[37] Gina Columbus. Expert Discusses Microsatellite Instability, Immunotherapy in CRC. http://www .onclive.com/web-exclusives/expert-discusses-microsatellite-instability-immunotherapy-in-crc 2016. [Online; accessed 2016-12-06].

[38] Steven A Rosenberg. Interleukin 2 for patients with renal cancer. Nature clinical practice. Oncology, 4(9):497, 2007. ISSN 1743-4262. doi: 10.1038/ncponc0926.

[39] Martin Reck, Delvys Rodríguez-Abreu, Andrew G. Robinson, Rina Hui, Tibor Csoszi, Andrea Fülöp, Maya Gottfried, Nir Peled, Ali Tafreshi, Sinead Cuffe, Mary O’Brien, Suman Rao, Katsuyuki Hotta, Melanie A. Leiby, Gregory M. Lubiniecki, Yue Shentu, Reshma Rangwala, and Julie R. Brahmer. Pembrolizumab versus Chemotherapy for PD-L1-Positive Non-Small-Cell Lung Cancer. New England Journal of Medicine, 375(19):1823-1833, 2016. ISSN 0028-4793. doi: 10.1056/NEJMoa1606774.

[40] Chris Fellner. Ipilimumab (Yervoy) Prolongs Survival In Advanced Melanoma. P\&T, 37(9):503-512, 2012. ISSN $1052-1372$.

[41] A Amin and RL White. Interleukin-2 in Renal Cell Carcinoma: A Has-Been or a Still-Viable Option? Journal of Kidney Cancer and VHL, 1(7):74-83, 2014. doi: 10.15586/jkcvhl.2014.18.

[42] M E Smith and W L Ford. The recirculating lymphocyte pool of the rat: a systematic description of the migratory behaviour of recirculating lymphocytes. Immunology, 49(1):83-94, 1983. ISSN 0019-2805.

[43] Michael H. Kershaw, Jennifer A. Westwood, Linda L. Parker, Gang Wang, Zelig Eshhar, Sharon A. Mavroukakis, Donald E. White, John R. Wunderlich, Silvana Canevari, Linda Rogers-Freezer, Clara C. Chen, James C. Yang, Steven A. Rosenberg, and Patrick Hwu. A Phase I Study on Adoptive Immunotherapy Using Gene-Modified T Cells for Ovarian Cancer. Clinical Cancer Research, 12(20):6106-6115, 2006. ISSN 1078-0432. doi: 10.1158/10780432.CCR-06-1183.

[44] Ana Caterina Parente-Pereira, Jerome Burnet, David Ellison, Julie Foster, David Marc Davies, Sjoukje Van der Stegen, Sophie Burbridge, Laura Chiapero-Stanke, Scott Wilkie, Stephen Mather, and John Maher. Trafficking of CAR-Engineered Human T Cells Following Regional or Systemic Adoptive Transfer in SCID Beige Mice. Journal of Clinical Immunology, 31(4):710-718, 2011. doi: 10.1007/s10875-011-9532-8.

[45] Tapani Lahtinen, Paavo Karjalainen, and Esko M. Alhava. Measurement of bone blood flow with a 133Xe washout method. European Journal of Nuclear Medicine, 4:437, 1979.

[46] Christoph Scheiermann, Julie Gibbs, Louise Ince, and Andrew Loudon. Clocking in to immunity. Nature Reviews Immunology, 18:423-437, 2018. doi: 10.1038/s41577-018-0008-4.

[47] Linan Wang, Ning Ma, Sachiko Okamoto, Yasunori Amaishi, Eiichi Sato, Naohiro Seo, Junichi Mineno, Kazutoh Takesako, Takuma Kato, and Hiroshi Shiku. Efficient tumor regression by adoptively transferred CEA-specific CAR-T cells associated with symptoms of mild cytokine release syndrome. OncoImmunology, 5(9):1-13, 2016. ISSN 2162402X. doi: 10.1080/2162402X.2016.1211218.

[48] Markus Chmielewski, Olga Hahn, Gunter Rappl, Michael Nowak, Ingo H. Schmidt-Wolf, Andreas A. Hombach, and Hinrich Abken. T cells that target carcinoembryonic antigen eradicate orthotopic pancreatic carcinomas without inducing autoimmune colitis in mice. Gastroenterology, 143(4):1095-1107.e2, 2012. ISSN 00165085. doi: 10.1053/j.gastro.2012.06.037.

[49] Shan-Shan Jiang, Yan Tang, Yao-Jun Zhang, D-Sheng Weng, Zhong-Guo Zhou, Ke Pan, Qiu-Zhong Pan, Qi-Jing Wang, Qing Liu, Jia He, Jing-Jing Zhao, Jiang Li, Min-Shan Chen, Alfred E Chang, Qiao Li, and Jian-Chuan Xia. A phase I clinical trial utilizing autologous tumor-infiltrating lymphocytes in patients with primary hepatocellular carcinoma. Oncotarget, 6(38):41339-49, 2015. ISSN 1949-2553. doi: 10.18632/oncotarget.5463. 
[50] M. H. Geukes Foppen, M. Donia, I. M. Svane, and J. B.A.G. Haanen. Tumor-infiltrating lymphocytes for the treatment of metastatic cancer. Molecular Oncology, 9(10):1918-1935, 2015. ISSN 18780261. doi: 10.1016/j.molonc.2015.10.018.

[51] Richard A Morgan, Mark E Dudley, John R Wunderlich, Marybeth S Hughes, James C Yang, Richard M Sherry, Richard E Royal, Suzanne L Topalian, Udai S Kammula, Nicholas P Restifo, Zhili Zheng, Azam Nahvi, Christiaan R De Vries, Linda J Rogers-freezer, Sharon A Mavroukakis, and Steven A Rosenberg. Cancer Regression in Patients. In Vitro, 126(2006):126-130, 2007. doi: 10.1126/science.1129003.

[52] Eric Tran, Paul F. Robbins, Yong-Chen Lu, Todd D. Prickett, Jared J. Gartner, Li Jia, Anna Pasetto, Zhili Zheng, Satyajit Ray, Eric M. Groh, Isaac R. Kriley, and Steven A. Rosenberg. T-Cell Transfer Therapy Targeting Mutant KRAS in Cancer. New England Journal of Medicine, 375(23):2255-2262, 2016. ISSN 0028-4793. doi: 10.1056/NEJMoa1609279.

[53] F. Kallinowski, K. H. Schlenger, S. Runkel, M. Kloes, M. Stohrer, P. Okunieff, and P. Vaupel. Blood Flow, Metabolism, Cellular Microenvironment, and Growth Rate of Human Tumor Xenografts. Cancer Res., 49(14): 3759-3764, 1989. ISSN 0008-5472.

[54] Cassandra Willyard. The mice with human tumours: Growing pains for a popular cancer model. Nature, 560: 156-157, 2018. doi: 10.1038/d41586-018-05890-8.

[55] Rebar N. Mohammed, H. Angharad Watson, Miriam Vigar, Julia Ohme, Amanda Thomson, Ian R. Humphreys, and Ann Ager. L-selectin Is Essential for Delivery of Activated CD8+ T Cells to Virus-Infected Organs for Protective Immunity. Cell Reports, 14(4):760-771, 2016. ISSN 22111247. doi: 10.1016/j.celrep.2015.12.090.

[56] David Masopust and Jason M. Schenkel. The integration of T cell migration, differentiation and function. Nat. Rev. Immunol., 13(5):309-320, May 2013. ISSN 1474-1733. doi: 10.1038/nri3442.

[57] Javier Mestas and Christopher C. W. Hughes. Of Mice and Not Men: Differences between Mouse and Human Immunology. J. Immunol., 172(5):2731-2738, Mar 2004. ISSN 0022-1767. doi: 10.4049/jimmunol.172.5.2731.

[58] Ruud P. M. Dings, Melissa Loren, Hanke Heun, Elizabeth McNiel, Arjan W. Griffioen, Kevin H. Mayo, and Robert J. Griffin. Scheduling of Radiation with Angiogenesis Inhibitors Anginex and Avastin Improves Therapeutic Outcome via Vessel Normalization. Clin. Cancer Res., 13(11):3395-3402, Jun 2007. ISSN 1078-0432. doi: 10.1158/10780432.CCR-06-2441.

[59] Limor Amit, Irit Ben-Aharon, Liat Vidal, Leonard Leibovici, and Salomon Stemmer. The Impact of Bevacizumab (Avastin) on Survival in Metastatic Solid Tumors - A Meta-Analysis and Systematic Review. PLoS One, 8(1): e51780, Jan 2013. ISSN 1932-6203. doi: 10.1371/journal.pone.0051780.

[60] Cor H.J. Lamers, Yarne Klaver, Jan W. Gratama, Stefan Sleijfer, and Reno Debets. Treatment of metastatic renal cell carcinoma (mRCC) with CAIX CAR-engineered T-cells-a completed study overview. Biochemical Society Transactions, 44(3):951-959, 2016. ISSN 0300-5127. doi: 10.1042/BST20160037. 



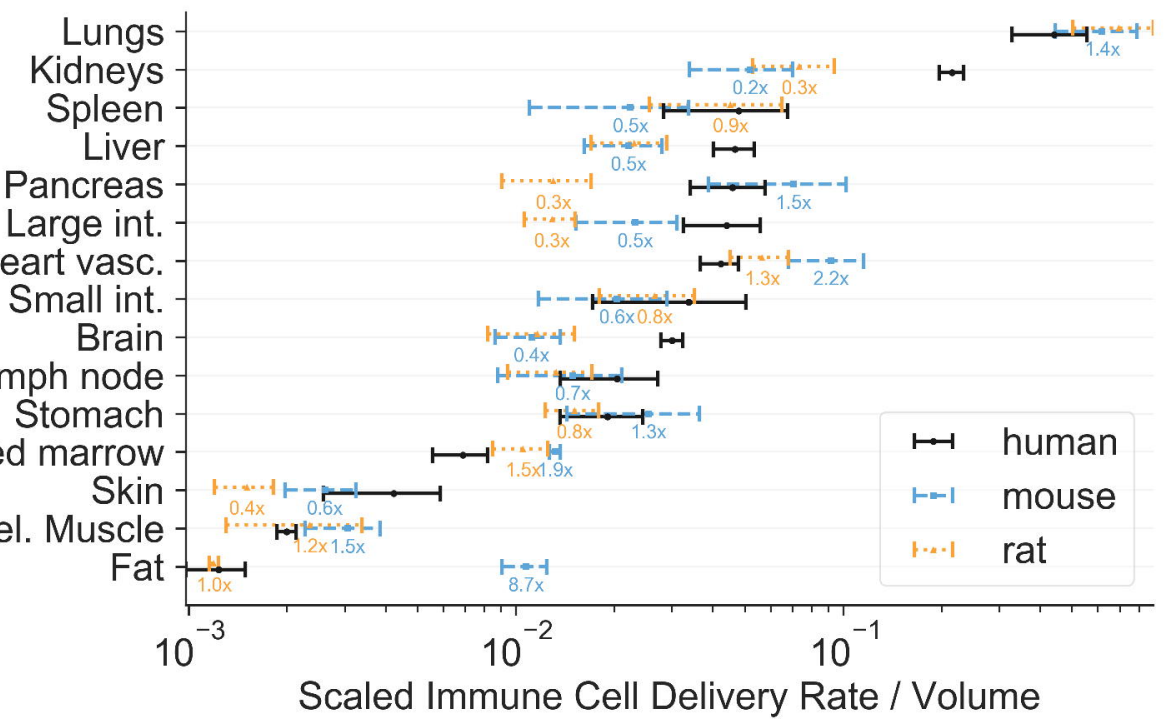




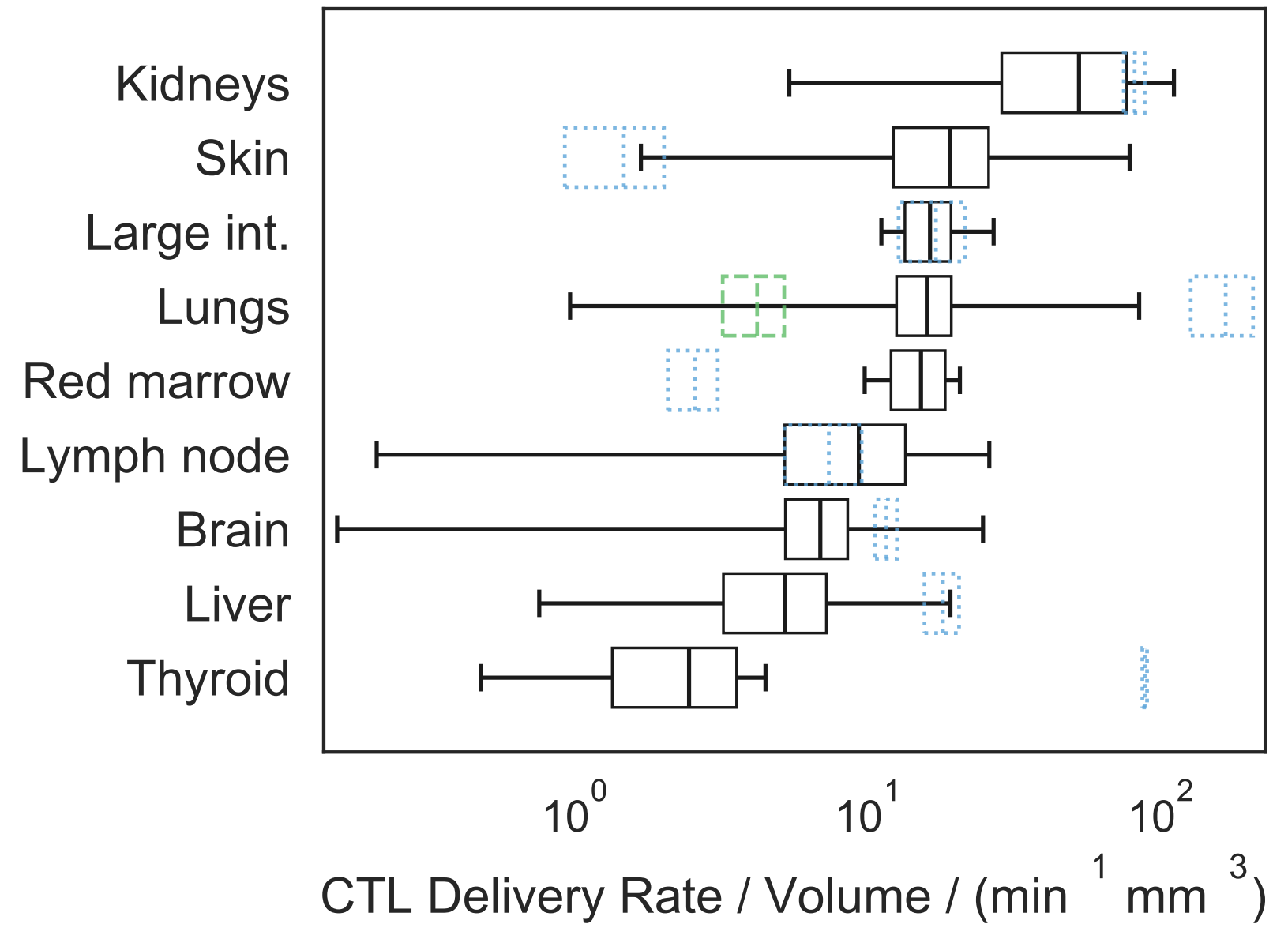


